\title{
Comparative Study of Robustness of Statistical Methods for Laboratory Proficiency Testing
}

\author{
Isao KoJIMA ${ }^{* \dagger}$ and Kazutoshi KaKITA** \\ *Japan Accreditation Board, 1-22-1 Higashi-gotanda, Shinagawa, Tokyo 141-0022, Japan \\ **Nippon Steel \& Sumikin Technology Co., Ltd., KSP-A101, 3-2-1 Sakado, Takatsu, Kawasaki 213-0012, Japan
}

\begin{abstract}
A Monte Carlo approach has been applied to investigate the statistical properties of data analysis methods for proficiency testing, namely, the median-nIQR, Q-method, Algorithm A and Classical method. Simulations were carried out by using a population consisting of two normal distributions, a main population without bias and a sub-population with bias. The difference for robustness among the methods has been discussed. An actual PT for a dioxin isomer was well interpreted by using the population model employed in this study.
\end{abstract}

Keywords Robustness, proficiency testing, Monte Carlo approach, median-nIQR, Algorithm A, Q-method

(Received August 3, 2014; Accepted October 27, 2014; Published December 10, 2014)

\section{Introduction}

Proficiency testing $(\mathrm{PT})^{1}$ as a means to evaluate the performance of testing laboratories plays an important role in laboratory accreditation by ISO/IEC $17025 .^{2}$ The $z$ score is usually used as a criteria to show the performance of the laboratory. In order to calculate the $z$ score, it is necessary to obtain the assigned value and the standard deviation for proficiency assessment (SDPA). However, a problem is that these values are affected by erroneous reports (data with biases, also often referred to as outliers). ISO/IEC 17043 requires the use of robust statistical methods in order to minimize the influence of such outliers on performance statistics, where robust statistical methods are defined as statistical methods that are not easily affected by small departures from underlying assumptions surrounding an underlying probabilistic model. ${ }^{1}$ In Annex $\mathrm{C}$ of ISO/DIS.2 13528, median-nIQR, Q-method and Algorithm A are described as robust methods. ${ }^{3}$ Although, there are some comparative studies between methods for individual $\mathrm{PT}^{4,5}$ an overall statistical comparison of the robustness of the methods has not been made so far.

In this report, the Monte Carlo approach has been applied to investigate the difference of the statistical properties among the methods described above. The distribution with bias was applied to the simulation. It was found that the robustness to outliers of the median-nIQR method was more significant than the other robust methods, such as the Algorithm A and the Q-method. An actual PT for a dioxin isomer was also discussed in comparison with the simulation.

\footnotetext{
† To whom correspondence should be addressed.

E-mail: Isao.Kojima@jab.or.jp
}

\section{Experimental}

\section{Calculation methods}

The $z$ score is an index indicating the performance of laboratories included in this study using the average value and the standardized deviation of the reported values. The $z$ score is given by the following equation,

$$
z=\frac{x-X}{\sigma_{\mathrm{pt}}}
$$

where, $X$ is called an assigned value of PT, and $\sigma_{\mathrm{pt}}$ is the SDPA. They are calculated for the statistical methods from A to E described below in this study.

The criteria of the performance of the laboratory is usually as follows: an absolute value of the score exceeding 3 indicates "unsatisfactory" performance, a value from 2 to 3 indicates "questionable" performance, and a value lower than 2 indicates "satisfactory" performance.

A. Reference method. The mean and the standard deviation of the main population are given as the assigned value and the SDPA, respectively.

B. median-nIQR method. The calculation algorithm is described in Annex C (normative) of ISO/DIS.2 13528. ${ }^{3}$ A median is used for the assigned value. When data is arranged in ascending order from the lowest value, the inter quartile range, IQR, is calculated from the difference between the 1 st and the $3 \mathrm{rd}$ quartiles. Then, normalize IQR, nIQR, given by $0.7413 \times \mathrm{IQR}$, is used as the SDPA.

C. Q-method. The calculation algorithm is described in Annex C (normative) of ISO/DIS.2 13528. ${ }^{3}$ This method requires more intense computational resources than others. Reported data are first converted into a new cumulative distribution relating to the distance of each data. The robust standard deviation is calculated from the inverse function of the cumulative distribution at a specific point. The assigned value is determined by the Hampel estimator. 


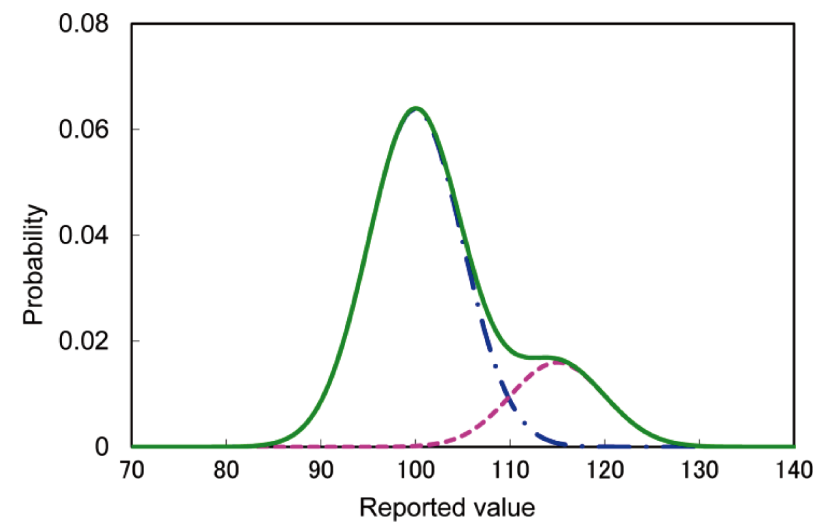

Fig. 1 Distribution of a population consisting of a main group and a sub-group. Solid dash line; main group, dashed line; sub-group, and solid line; total (sum of the two groups).

D. Algorithm A method. The calculation algorithm is described in Annex C (normative) of ISO/DIS.2 13528. ${ }^{3}$ In this method, measurement values outside of 1.5 times the robust standard deviation, $\mathrm{s}^{*}$, are replaced by the value at $1.5 \mathrm{~s}^{*}$, and then the robust mean and the standard deviation are calculated again. Iterative calculations by a computer is required.

E. Classical/Grubbs method. The multiple Grubbs test at the confidence level of $95 \%$ is carried out first to remove outliers. Then, the mean and the standard deviation are calculated from the rest of the data.

The calculation program for the Monte Carlo simulation was coded in EXCEL VBA language. A random number of the normal distribution was created by the Box-Muller method with an internal Rnd() function in VBA.

\section{Population model}

The population of the reported data of PT was assumed to contain two groups with a normal distribution with the same standard deviation, $\sigma$. One is the main group which reports results by performing correct measurement procedures, however, the other is the sub-group of smaller size which reports results with biases due to erroneous procedures. Here, the bias is equal to the difference in the average values of the two groups. Figure 1 shows an example of the distribution of a population, consisting of the main group of $80 \%$ and the sub group of $20 \%$, where the bias (called sub-group bias later) is three times the standard deviation. The mean and the standard deviation of the main group were given as 100 and 5, respectively. A subpeak is clearly observed at this sub-group bias.

\section{Results}

Changes in assigned values and SDPAs with group biases

Simulation was done for all 200 participating laboratories; 160 laboratories from a main group and 40 laboratories from a sub-group. Sampling was repeated 5000 times, and the assigned values and SDPAs were calculated for each sampling. The average values of the assigned values and SDPAs are shown in Figs. 2(a) and 2(b), respectively.

Of course, the average of assigned values is constant at 100 for the Reference method. As shown in Fig. 2(a), the assigned values of the median-nIQR method increase when increasing the sub-group bias up to $2 \sigma$ and become almost constant above $2 \sigma$. The Q-method shows a little larger increase to reach a maximum at around $4 \sigma$, and decreases to the reference value at
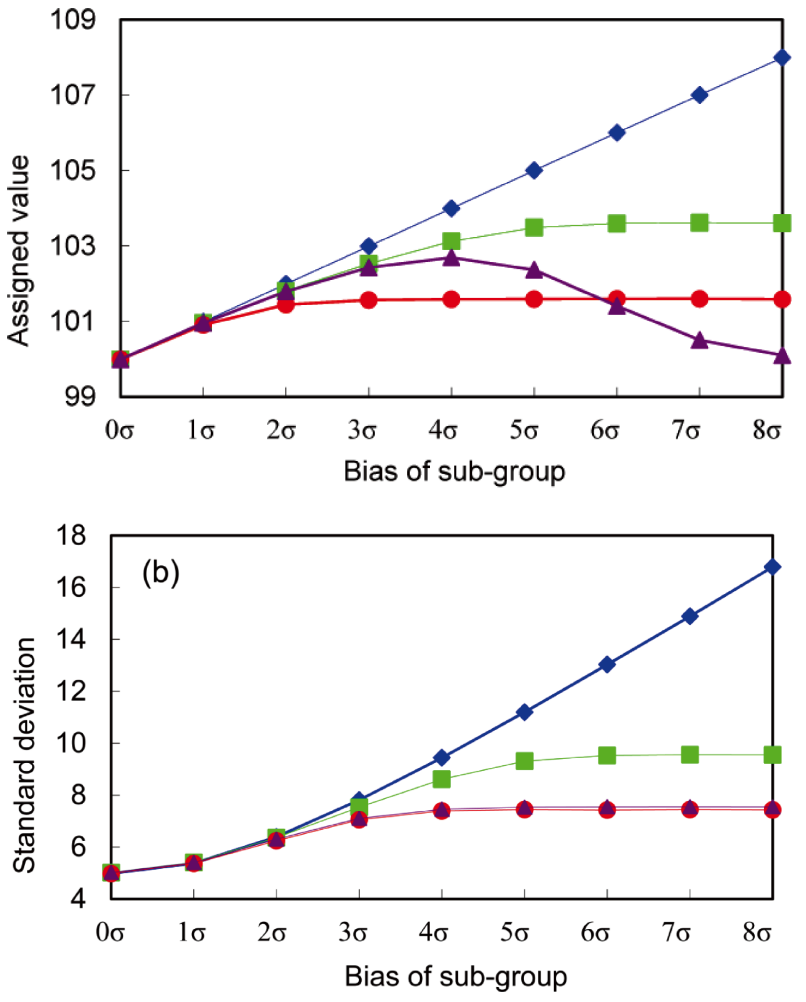

Fig. 2 Changes in: (a) the assigned value, and (b) the standard deviation of performance evaluation against the sub-group bias.

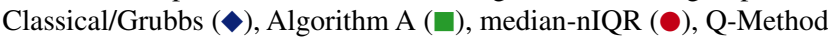
$(\Delta)$.

around $8 \sigma$. The Algorithm A method shows an increase similar to the Q-method up to $4 \sigma$, and reaches an almost constant value above $5 \sigma$. It should be noted that the median-nIQR method gives relatively closer values to the reference value than the Q-method up to $5 \sigma$ and than the Algorithm A method at any sub-group bias. The Classical method shows a constant and the largest increase with the sub-group bias. This is because almost no outlier is detected for the large size sub-group of $20 \%$ even by using the multiple Grubbs test.

The averaged values of the standard deviation show the features as given in Fig. 2(b). The standard deviation of the Reference method (not shown) is constant at 5. The closeness of each method to the Reference method is in the order of median-nIQR, Q-method, Algorithm A, and Classical, which is the same as in the case of the mean of assigned values except the Q-method does not show any decrease above $5 \sigma$.

\section{Difference of z scores to statistical methods}

In each repetitive calculation, the $z$ scores were also obtained. Figures. 3(a) and 3(b) show the changes in percentage of laboratories for which the $z$ scores were calculated to be larger than 3 and 2, respectively.

The percentages were found to increase when increasing the sub-group bias in all cases. In Fig. 4(a), the percentage of the Reference method is about $0.3 \%$ at the zero sub-group bias since all laboratories belong to a single normal distribution. Also, above the sub-group bias of $5 \sigma$, the percentage almost reaches the limit of $20 \%$ where almost all the laboratories of the sub-group were detected by the $z$ score. The percentages of the median-nIQR, Q-method and Algorithm A methods also increase with bias, although their increases are smaller than the Reference method. At around the sub-group bias of $5 \sigma$, the 
median-nIQR and the Q-method have larger percentages than the Algorithm A method. The percentages of the two methods
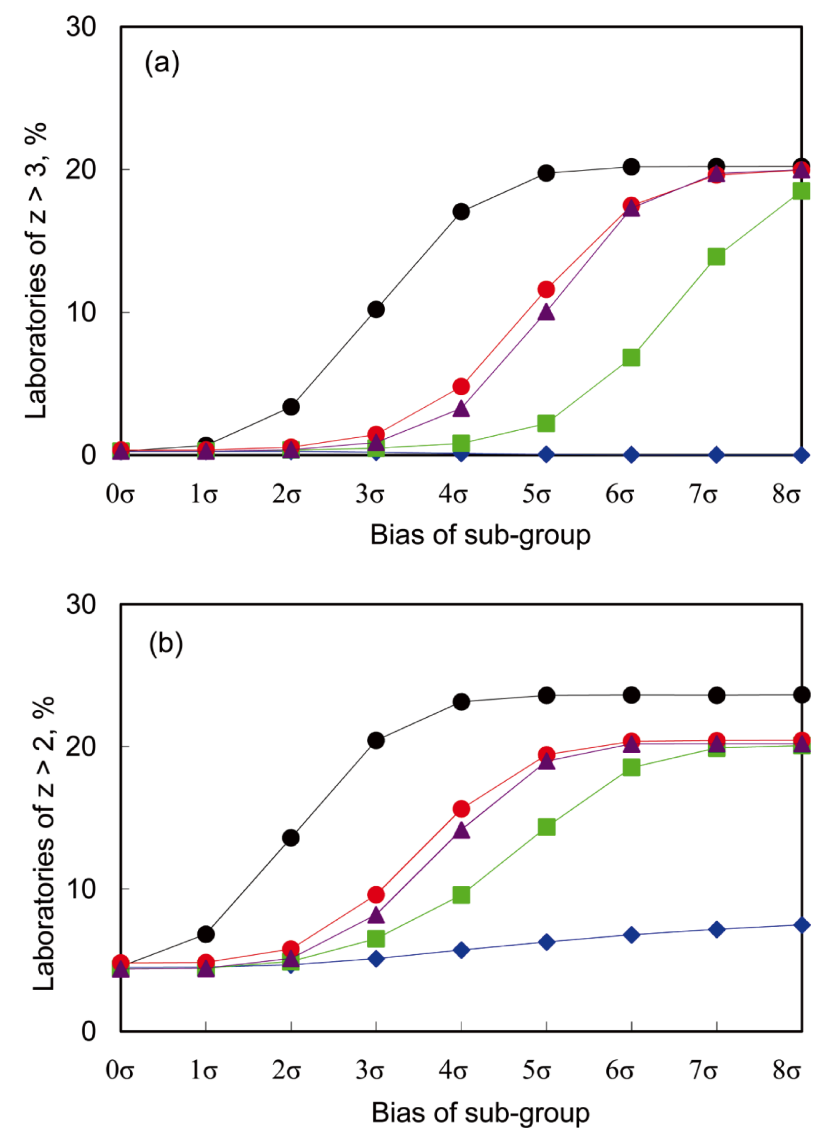

Fig. 3 Relationship between the sub-group bias and the percentage of laboratories with $z$ score $>3$ (a) and $z$ score $>2$ (b). Reference (ט),

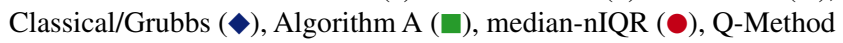
$(\Delta)$. reach $20 \%$ at more than $8 \sigma$. However, the percentage of the Classical method does not increase with bias. This is due to the same reason as in Fig. 2(a), that is, the Grubbs test is not effective. Figure 3(b) shows almost the same features as in Fig. 3(a) although the percentages shift to about $5 \%$ at zero sub-group bias due to the general rule for a normal distribution.

It should be noted again that the median-nIQR method gives relatively closer values to the reference value than the other methods in all ranges of sub-group biases.

\section{Effect of limited volume of data for statistical efficiency}

Effect of the sample size (here, the number of participant laboratories) is given as efficiency in statistics where a more efficient test needs fewer samples than a less efficient one to achieve a given performance. In order to investigate such effect, an additional calculation was performed to obtain the parameter called the degree of freedom.

The degree of freedom of an average of $\mathrm{N}$ data sampled from a normal distribution is known to be $\mathrm{N}-1$. An additional statistical treatment by robust methods may lead to some decrease in the degree of freedom. Generally, a larger degree of freedom is desirable to attain a smaller variance of a calculated statistical parameter. Here, Eq. $(2)^{6}$ is applied to estimate the effective degrees of freedom for averages of various robust methods, where $\bar{s}(x)$ and $s(\bar{s}(x))$ are the mean of standard deviation and the standard deviation of the standard deviation for the corresponding robust calculation, respectively.

$$
v \approx \frac{1}{2}\left[\frac{s(\bar{s}(x))}{\bar{s}(x)}\right]^{-2}
$$

Calculated results are given in Table 1 where the number of laboratories was varried from 20 to 500 and the Monte Carlo sampling was conducted 30000 times from a single normal distribution. The obtained effective degrees of freedom decreased in the order of Classical $>$ Algorithm $\mathrm{A} \fallingdotseq \mathrm{Q}-$ Method $>>$ median-nIQR. Relative changes in the effective degrees of freedom for each method are almost constant for the number of

$2,3^{\prime}, 4,4^{\prime}, 5-\mathrm{PeCB}(\# 118)$

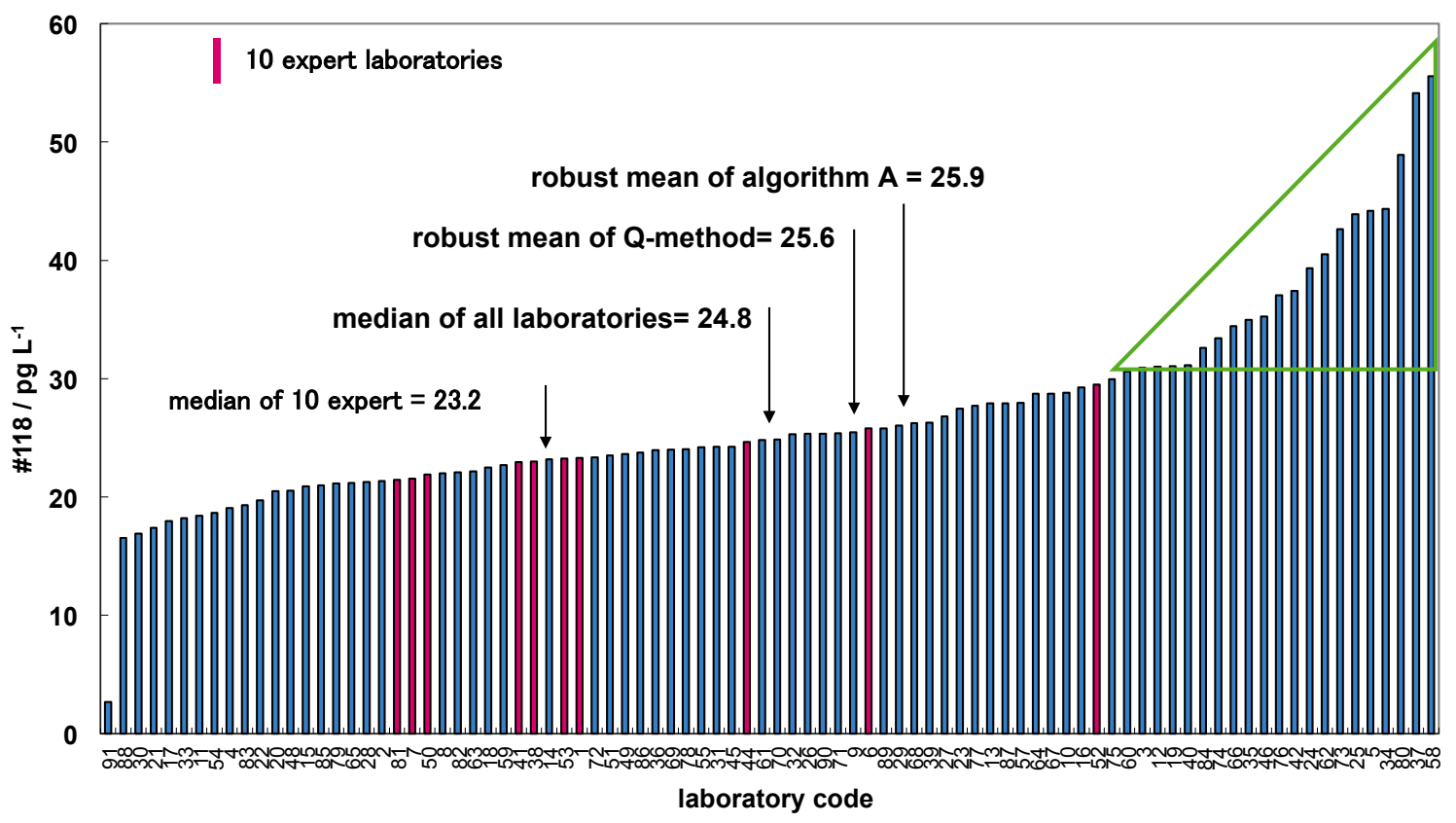

Fig. 4 An example of proficiency testing of the dioxin isomer \#118 (reproduced from the report of PT). 
Table 1 Effective degrees of freedom of statistical methods

\begin{tabular}{ccccc} 
Number of data & Median-nIQR & Q-Method & Algorithm A & Classical \\
\hline 20 & 7.6 & 12 & 14 & 19 \\
50 & 19 & 35 & 37 & 49 \\
100 & 37 & 77 & 72 & 99 \\
200 & 74 & 158 & 140 & 190 \\
500 & 180 & 405 & 360 & 490 \\
\hline
\end{tabular}

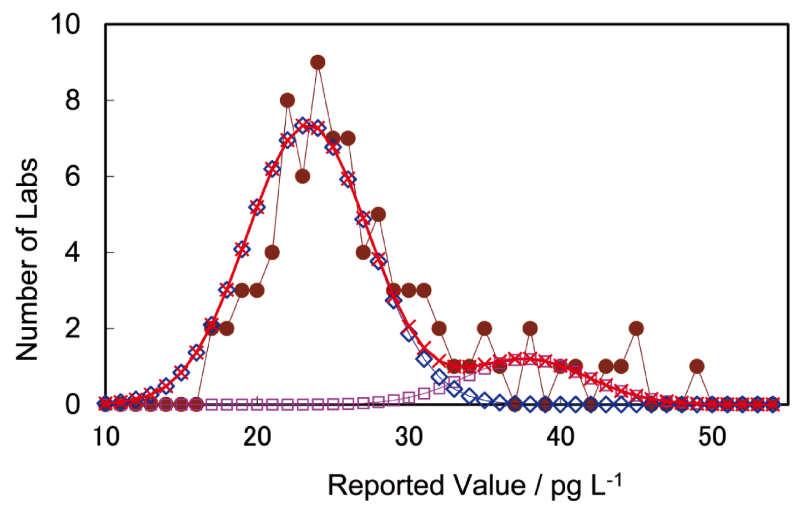

Fig. 5 The best fitting to two normal distributions of data of the dioxin proficiency testing given in Fig. 4. Reported data (๑), subgroup ( $\square)$, main group $(\diamond)$, and total $(\mathbf{X})$.

data. In calculating the $z$ score, consideration should be given to the fact that a small degree of freedom causes a large scattering of evaluated data for PT and may result in an erroneous interpretation especially for the median-nIQR method as it shows the smallest degrees of freedom of about $38 \%$ of a sample size.

\section{Comparison with PT results for a dioxin isomer}

Figure 4 shows the results of the determination of \#118 (dioxin-like polychlorinatedbiphenyls, 2,3',4,4',5-PeCB) isomer in the 12th dioxin PT of simulated wastewater containing dioxin, conducted by the Japan Society for Analytical Chemistry. ${ }^{7}$ The reported data of 88 laboratories are ranked according to values. The 10 laboratories that participated in previous similar comparisons were designated as expert laboratories, and their results were evaluated by median-nIQR. The sequence of the assigned values calculated by several methods was as follows: The median of the expert laboratories was less than the median of all laboratories, which was less than the robust means of the Q-method and Algorithm A method. For this PT, a positive bias was expected because the analyzed result tended to increase when testing rooms were contaminated by the analyte (\#118 isomer). Laboratories that might have positive biases are shown in the triangle area of the right upper side in the figure. Assigning the median of expert laboratories to the assigned value of the Reference approach, the sequence of the four methods (Reference method, median-nIQR, Q-method and Algorithm A) for the dioxin PT, in which smaller number (equal to 88) of laboratories participated, is consistent with the sequence of the simulated results for 200 laboratories for the distribution with a sub-group bias as described before .

The results of the dioxin PT was fitted to the distribution model of the population used in this study. That is, the results were fitted to the two normal functions with the same standard deviation, where optimization was performed by using EXCEL solver. Due to the limited number of participant laboratories, the distribution of the group with a sub-group bias was reproduced incompletely; however, it nevertheless effectively represents the structural feature of distribution obtained by the simulation. The calculated distribution shows the sub-group with size of $14 \%$ and with the sub-group bias as 3.6 times the standard deviation. The percentage of laboratories with $z$ score $>$ 3 is estimated at about $9 \%$ by using the median-nIQR method, which is a little larger than that expected from Fig. 3(a). This is because the larger scattering of biased data leads to an underestimate of the mean of the sub-group.

\section{Discussion}

Based on the simulation results, the differences in robustness between the methods are discussed. The Reference method is almost equivalent to the formulation to use a certified reference value described in ISO/DIS. $213528,{ }^{3}$ and will yield the best decision that can be reached statistically. Therefore, the closeness to the Reference method of each method implies its higher robustness. The order of the closeness, median-nIQR, Q-method, Algorithm A, and Classical method, as shown in Figs. 2 and 3 suggests that the median-nIQR method exhibits the highest robustness to outliers among the three robust methods.

The sampling number of 200 was chosen because the standard deviations are almost consistent between the methods when the number of laboratories is more than 200. The averages of 5000 Monte Carlo simulations shown in Fig. 2 are not much influenced by the number of each sampling. So, the trend of robust methods obtained in the assigned values and in the $z$ scores does not change, even if the number of samplings for each simulation is reduced to 20 .

However, the median-nIQR results do not always give the best conclusion in the actual PT. The reduction of degrees of freedom causes a statistical increase in the variation of each PT and should lead to a higher probability of reaching an erroneous outcome. This is because a large part of data of $50 \%$ is excluded for the median-nIQR in the calculation of the assigned value and SDPA. Therefore, both the factors of robustness to outliers and the effective degree of freedom should be considered in order to avoid erroneous interpretations. It would sometimes be better, especially in the case of a smaller number of participant laboratories, to simply employ the Classical method with large efficiency or to use it combined with the median-nIQR method with better robustness.

\section{References}

1. ISO/IEC 17043, "Conformity assessment - General requirements for proficiency testing".

2. ISO/IEC 17025, "General requirements for the competence of testing and calibration laboratories".

3. ISO/DIS.2 13528, "Statistical methods for use in proficiency testing by interlaboratory comparisons".

4. S. Jaroslava and Z. Jiri, Accredit. Qual. Assur., 2009, 14, 467.

5. R. Pedro, L. M. Jose, and M. S. Jose, Accredit. Qual. Assur., 2008, 13, 493.

6. ISO/IEC Guide 98-3, "Guide to the expression of uncertainty in measurement, GUM, 1995 with minor modifications".

7. The 12th proficiency testing for dioxin analysis, the Japan Society for Analytical Chemistry, 2010. 